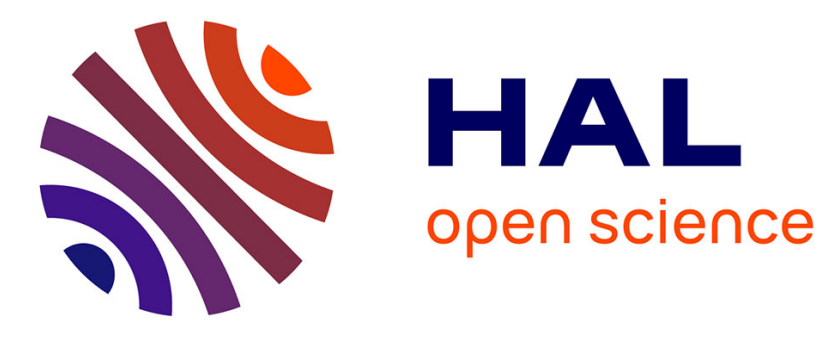

\title{
Mesure des moments magnétiques. Applications spatiales
}

\author{
J. Chalvet
}

\section{To cite this version:}

J. Chalvet. Mesure des moments magnétiques. Applications spatiales. Revue de Physique Appliquée, 1970, 5 (1), pp.72-76. 10.1051/rphysap:019700050107200 . jpa-00243377

\section{HAL Id: jpa-00243377 https://hal.science/jpa-00243377}

Submitted on 1 Jan 1970

HAL is a multi-disciplinary open access archive for the deposit and dissemination of scientific research documents, whether they are published or not. The documents may come from teaching and research institutions in France or abroad, or from public or private research centers.
L'archive ouverte pluridisciplinaire HAL, est destinée au dépôt et à la diffusion de documents scientifiques de niveau recherche, publiés ou non, émanant des établissements d'enseignement et de recherche français ou étrangers, des laboratoires publics ou privés. 


\title{
MESURE DES MOMENTS MAGNÉTIQUES. APPLIGATIONS SPATIALES
}

\author{
Par J. CHALVET, \\ Compagnie des Compteurs (C.d.G.), Centre d'Électronique, Massy (France).
}

\begin{abstract}
Résumé. - Équivalant magnétiquement à un doublet, tout satellite gravitant dans un milieu caractérisé par son induction magnétique propre est soumis, en conséquence, à un couple. Ce couple exerce sur l'attitude du satellite soit un rôle utile de commande dans le cas d'une stabilisation magnétique, soit un rôle parasite de dépointage progressif pour tout autre mode de stabilisation. Aussi s'avère-t-il indispensable, pour l'évaluation prévisionnelle des mouvements angulaires du satellite, de déterminer complètement le moment magnétique vectoriel.

Cette détermination nécessite une expérimentation au sol s'effectuant en milieu simulé reproduisant, aussi fidèlement que possible, les conditions d'ambiance magnétique orbitales. Compte tenu de cet impératif, la méthode de mesure appropriée permettant de lever tous les doutes fait alors avantageusement appel à une série de données magnétométriques. La méthode repose sur un traitement simple des signatures magnétiques qu'accusent deux sondes orthogonales disposées à distance suffisante du satellite lorsque l'on soumet ce dernier à des mouvements tant de translation que de rotation sur lui-même; toutefois, la procédure par translation ne s'impose que dans le cas d'une aimantation induite notable, soit pour des satellites gravitant sur orbites de faibles apogées.

Mise en œuvre sur les satellites français FR1 et D1 et sur les satellites européens ESRO1 et HEOS, la méthode magnétométrique s'est avérée dans l'ensemble satisfaisante, les résultats obtenus se centrant en moyenne sur une valeur de moment assez précise ; une dispersion notable est cependant à souligner du fait du parasitage qui affecte les faibles niveaux magnétiques détectés.
\end{abstract}

Abstract. - As it is known that a satellite is magnetically equivalent to a dipole, every satellite what is orbiting in magnetic space will be subject to a torquing-effect. This torque can be used to set-up the attitude of the satellite. In other cases a perturbation should be observed in the attitude produced by this magnetic effect. Consequently, by means of a measurement of the over-all vectorial magnetic moment, a computation of the corresponding attitude variations is possible.

This measurement will be performed in a magnetic space-simulation laboratory. The magnetic measurement technique is based upon a recording of datas transmitted by rectangular magnetic sensors fixed enough far away of the rotating satellite. In case of satellite orbiting near Earth's surface a translation of the satellite will be necessary to measure the magnetic moment inducted.

This method has been successfully applied for European Satellites FR1, D1, ESRO1 et HEOS. Measurements corresponding in weak magnetic field are affected by external perturbations ; consequently a large number of measures is required.

1. Généralités. - Pour caractériser l'aimantation d'un objet, un satellite notamment, on a recours à un modèle mathématique constitué d'une série de pôles magnétiques, opposés en signe deux à deux. Comme deux pôles symétriques sont toujours sensiblement concentrés en un même point, le barycentre des masses magnétiques positives est très voisin du barycentre négatif.

Le modèle barycentrique dipolaire résultant, encore dénommé doublet équivalent, ne se justifie que pour les évaluations d'influence magnétique globale, telles que couple magnétique ou répartition des champs induits à grande distance. La mesure du moment magnétique équivalent, défini comme le produit de la masse magnétique barycentrique par la distance séparant les deux pôles du doublet, fera donc appel, soit à une procédure dynamique mettant en jeu le couple magnétique, soit à une procédure statique s'appuyant sur l'analyse de la répartition des champs externes à distance suffisante. Les méthodes dynamiques dressent toutes un bilan énergétique relatif à un mouvement de rotation de l'objet; comme dans de tels bilans, seuls des couples axiaux entrent en jeu, ces méthodes ont donc le défaut de laisser indéterminée la position du doublet équivalent. Tel n'est pas le cas de la procédure statique d'analyse de la répartition des champs externes. Cette répartition obéit à une loi de décroissance simple en inverse cube de la distance, à condition que l'observation porte sur des distances supérieures à deux fois et demie la plus grande dimen- 
sion de l'objet. Dans ces conditions, les lois de cause à effet étant cubiques, 18 points de mesure suffisent pour déterminer les six paramètres de définition du vecteur moment magnétique qui sont, classiquement, les trois coordonnées cartésiennes de position, les deux paramètres angulaires de direction et le module; pour lever toute ambiguïté sur les paramètres angulaires et sur les signes, il faut en fait disposer des trois composantes de champ qu'accusent trois sondes trirectangles en chacun des 18 points, soit 54 mesures en tout. Étant donné les erreurs inévitables de mesures, les limites de validité de l'approximation dipolaire et la complexité de la résolution mathématique, la précision finale est sujette à caution; aussi la solution consiste alors à multiplier les mesures, puis à effectuer un traitement statistique des différents résultats.

Insuffisance ou complexité trop grande sont les défauts respectifs affectant les méthodes dynamiques et statiques. On peut y remédier par une procédure de compromis qui va retenir notre attention.

2. Méthode de résolution magnéto-dynamique. La méthode proposée s'appuie sur l'observation des signatures magnétiques qu'accusent des sondes détectrices lorsque le satellite est animé de mouvements de rotation et même éventuellement de mouvements de translation. De la confrontation des signatures en présence pour différentes conditions d'observation, on déduit les signatures significatives; traitées judicieusement, ces dernières permettent la détermination simple des caractéristiques du moment magnétique cherché. La procédure retenue se décompose en étapes successives :

- détermination de la position du doublet permanent,

- obtention de la composante verticale du moment magnétique permanent,

- obtention, tant en module qu'en direction, de la composante horizontale du moment permanent,

- mêmes opérations en ce qui concerne le moment magnétique induit dans une ambiance orbitale.

2.1. Positionnement du Doublet Permanent. Disposons une sonde verticalement, à distance suffisante du satellite en rotation dans une ambiance amagnétique. Considérant les composantes horizontales et verticales du doublet permanent, la signature accusée par la sonde sera généralement fonction de ces deux composantes. Toutefois, si la sonde est à la cote du doublet, on s'affranchit alors du dipôle horizontal à contribution dissymétrique vu sa dissymétrie directionnelle lors de la rotation, si bien que le critère de définition de la cote du doublet implique l'obtention d'une signature symétrique de part et d'autre des extréma déphasés naturellement entre eux d'un angle $\pi$.

L'amplitude et le repérage angulaire de chaque extrémum par rapport à l'axe de symétrie horizontal d'observation définit simplement le rayon vecteur du doublet en coordonnées polaires, soit respectivement son module et sa direction.

2.2. DÉtermination DU DOUblet Vertical PERMANENT. - Prenant pour plan de figure le plan horizontal contenant le doublet en rotation assujetti à un mouvement circulaire, le schéma suivant caractérise alors l'observation.

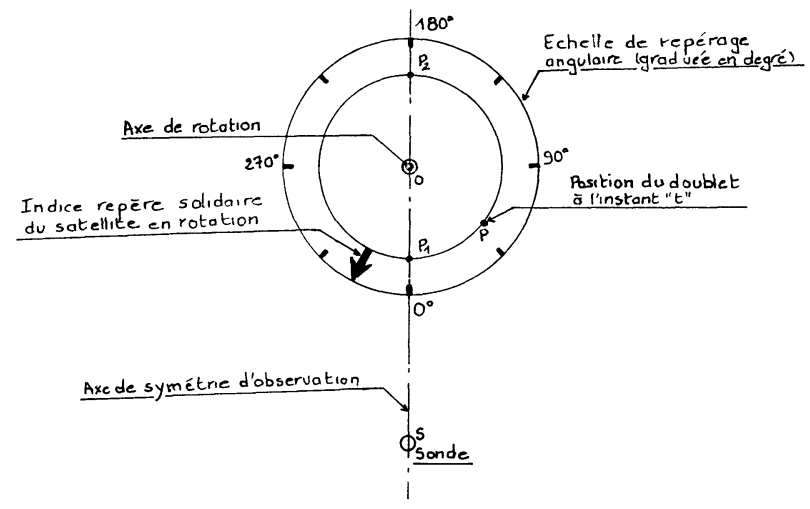

Désignant par « $r$ » le rayon de giration du doublet OP, par « $d »$ la distance d'observation OS, les extréma en $\mathrm{P}_{1}$ et $\mathrm{P}_{2}$ obéissent aux relations respectives suivantes :

$$
H_{\mathrm{P}_{1}}=\frac{M_{v}}{(d-r)^{3}} ; \quad H_{\mathrm{P}_{2}}=\frac{M_{v}}{(d+r)^{3}} .
$$

De la connaissance des amplitudes de champ extrêmes $H_{\mathrm{P}_{1}}, H_{\mathrm{P}_{2}}$ et de la distance « $d$ », on déduit simplement le rayon de giration « $r$ » et le module « $M_{n}$, du moment magnétique du doublet vertical.

2.3. ObTention du doublet horizontal PeRmaNENT. - Soumettons le satellite à une rotation autour de l'axe vertical du doublet déterminé précédemment. Une sonde magnétométrique disposée horizontalement à distance « $d$ » suffisante et visant le doublet accuse alors une signature dont l'amplitude crête à crête « $\Delta H »$ définit le moment cherché par la relation :

$$
M_{H}=\frac{\Delta H \times d^{3}}{4} \text {. }
$$

Le repérage angulaire des extréma fixe d'autre part la direction du doublet.

\subsection{DÉtermination DU DOUBLET VeRTIGAL INDUIT.} - Simulons l'ambiance magnétique orbitale dans laquelle gravite le satellite. En disposant verticalement une sonde à la cote du doublet induit, on s'affranchit du doublet horizontal correspondant variable en rotation. Le critère précédent peut s'appliquer dans ce cas mais sur la signature différence (permanent + induit) d'une part, permanent d'autre part. Comme l'induit horizontal présente une signature périodique harmonique 2 de la fréquence de rotation, on peut tenir compte de cette propriété pour alléger au mieux le dépouillement. Assez fréquemment, d'ailleurs, les dipôles permanents et induits sont sensiblement confondus, d'où un dépouillement simplifié.

2.5. Obtention DU DOUBlet hoRizontal induit. - Comme la loi de variation en rotation du moment horizontal induit n'est généralement pas connue, on soumet le satellite à un mouvement de translation. Disposée selon le Nord-Sud magnétique et située à la cote du doublet induit, une sonde accuse dans ces conditions une signature résultant de la contribution des moments permanents et de l'induit horizontal. En soustrayant la contribution du moment permanent expérimentalement ou par calcul, on déduit par suite la signature relative au moment induit seul axé Nord-Sud. 
Pour trouver l'amplitude du moment et sa direction, on doit se référer à deux points de la courbe, les extréma par exemple.

2.6. Limitation de la mÉthode. - A la suite de cet exposé de principe, il est bon de souligner quelques remarques relatives aux difficultés liées intrinsèquement à la méthode.

Ces difficultés tiennent aux faibles amplitudes mises en jeu par les signatures; aussi faut-il s'affranchir de toutes causes perturbatrices telles que variation du champ terrestre durant l'observation et influence au niveau du capteur du dispositif de simulation d'ambiance. En ce qui concerne les variations locales du champ terrestre, on les compense en disposant en montage différentiel sur le capteur de mesures un autre capteur de référence également affecté par ces variations et par elles seules. En dérivant une faible fraction du courant primaire de simulation sur un petit solénoïde de compensation entourant le capteur, on s'affranchit d'autre part de l'influence du dispositif simulateur; mais on doit noter qu'à chaque déplacement de la sonde détectrice, il faut retoucher le réglage de compensation.

3. Moyens mis en œuvre par la méthode. - Avant de donner quelques applications, énumérons l'appareillage que nécessite la méthode :

- Un dispositif de simulation d'ambiance, se présentant avantageusement sous forme d'une configuration bi-axiale de cadres rectangulaires compensant respectivement les composantes horizontales et verticales du champ terrestre. En dehors de l'obtention d'un volume d'homogénéité important dans le sens de la longueur, le choix d'une configuration rectangulaire s'impose du fait de la mise en translation de l'objet test, satellite ou autre;

- Un dispositif de détection, voire deux, compensé des différentes sources de parasitage et réglable en position;

- Des moyens mécaniques permettant en plus d'un mouvement de translation une mise en rotation aisée du satellite ou de tout autre objet autour d'un axe vertical quelconque;

- Enfin un système approprié de repérage angulaire et de distance.

4. Applications spatiales. - Diverses applications sont centrées sur le moment magnétique. Dans le domaine spatial, on peut jouer sur ce paramètre de base à l'échelle d'un satellite en agissant sur les caractéristiques magnétiques et l'implantation des sous-ensembles à intégrer. L'exemple type d'application d'une telle procédure, dans le but de réduire le moment magnétique global, donc l'influence parasite s'exerçant sur des sondes magnétométriques montées à bord, est fourni par le satellite européen HEOS A; les tests magnétiques préliminaires, dont $M$. Vuye a dressé un récapitulatif dans son exposé, servent d'éléments de référence pour définir la meilleure implantation. De toute manière, il est loisible de concevoir, à la limite, une compensation passive à distance du parasitage magnétique par intégration d'aimants dont les caractéristiques s'opposent au moment propre du satellite; soulignons que, pour une telle opération, il est impératif de disposer de la position du doublet magnétique équivalent, d'où l'intérêt de la méthode détaillée dans cet exposé.

En possession du moment magnétique résultant, l'application primordiale concerne l'évaluation du couple s'exerçant sur un satellite en gravitation. Le milieu orbital étant caractérisé par une induction B, le couple en question $\mathbf{M} \wedge \mathbf{B}$ peut jouer soit un rôle utile de commande d'attitude dans le cas d'une stabilisation magnétique, soit plus généralement un rôle parasite de dépointage progressif pour tout autre mode de stabilisation. Sur les exemples qui suivent, nous allons illustrer ces deux aspects en précisant les ordres de grandeur des paramètres magnétiques.

Dans le cas du projet du satellite franco-allemand Symphonie, à orbite fortement excentrée, le moment magnétique équatorial ne devra pas excéder $3,5 \mathrm{~A} . \mathrm{m}^{2}$, soit 3500 unités u.e.m. G.G.S., sous peine d'agir d'une manière néfaste sur l'attitude. Dans le cas du satellite français D1, de petites dimensions et à faible apogée, le moment magnétique se situait aux environs de 120 G.G.S. ou $0,12 \mathrm{~A} . \mathrm{m}^{2}$; les problèmes d'attitude n'offraient pas, dans ce cas, un caractère critique, les couples magnétiques mis ainsi en jeu restant dans des limites tolérables.

Pour conclure, nous considérerons le cas particulièrement intéressant du satellite européen ESRO1 stabilisé magnétiquement à l'aide de deux forts aimants disposés selon son axe. Véritable boussole, le satellite s'oriente alors suivant le vecteur champ terrestre; mais comme l'orbite est polaire, le vecteur champ en question varie continuellement lors du mouvement de gravitation du satellite. L'axe de celui-ci est soumis en conséquence à un mouvement oscillatoire dont on désire limiter le débattement angulaire à $10^{\circ}$. Dans ce but, on dispose d'une configuration de 20 barreaux en mu-métal en forme de cage, chaque face comprenant cinq barreaux équidistants entre eux. La configuration étant disposée orthogonalement par rapport aux aimants, sous l'effet combiné d'un dépointage et de la rotation propre du satellite, chacun d'entre eux est par suite soumis à un champ magnétique variable et périodique d'amplitude He. La configuration présente donc, du fait de l'hystérésis magnétique, une dissipation d'énergie freinant le dépointage. Les pertes d'une particule élémentaire s'expriment simplement, en unités G.G.S., par la loi de Warburg : $\oint H_{\mathrm{e}} d M$. La formule précédente s'applique au cas d'un barreau pour un cycle complet. Dans ce cas, en effet, le champ démagnétisant « $H_{\mathrm{d}}$ » caractéristique de l'influence magnétique mutuelle entre particules est proportionnel à chaque instant au moment magnétique global du barreau, si bien que ce champ ne donne pas de pertes sur un cycle, la courbe $M\left(H_{\mathrm{d}}\right)$ linéaire étant naturellement réversible, au contraire de son homologue $M\left(H_{\mathrm{e}}\right)$. On admet l'extension de cette propriété à la configuration en cage, donc une contribution du démagnétisant nulle sur un cycle. Les moments magnétiques étant déterminés conformément à la méthode décrite dans cet exposé, le tracé de la courbe $M\left(H_{\mathrm{e}}\right)$ pour un barreau et pour la configuration entière mène à des pertes d'ensemble égales à 15,5 fois environ la perte d'un barreau unique et non le coefficient 20 , comme un raisonnement simpliste pourrait le laisser supposer. 
Comme illustration de cette communication, nous présentons en annexe trois figures.

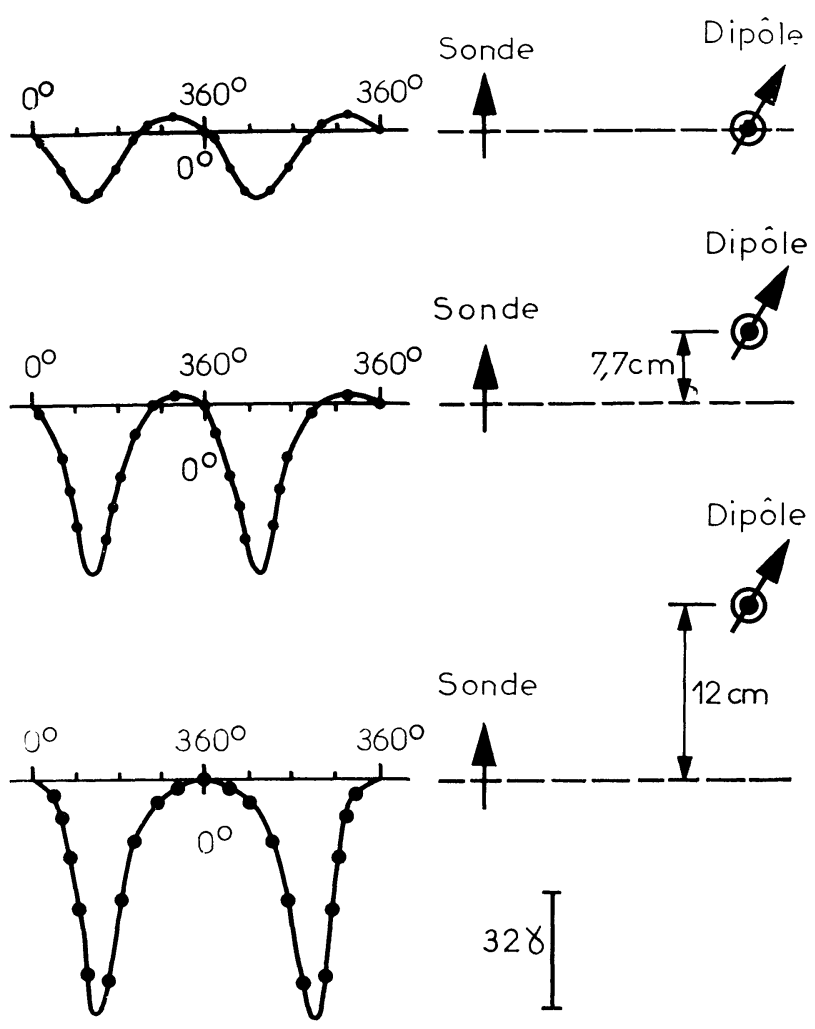

FIG. 1.

La figure 1 montre la variation des signatures verticales d'un aimant en fonction de la cote. Les caractéristiques expérimentales sont les suivantes :

Aimant $\quad: \varnothing, 1,5 \mathrm{~cm} ; L=12 \mathrm{~cm}$; incliné de $10^{\circ}$ sur l'horizontale

Rayon de giration : $r=20 \mathrm{~cm}$

Distance d'observa-

$$
\text { tion } \quad: \text { OS }=d=112 \mathrm{~cm}
$$

L'augmentation sensible de l'amplitude crête à crête des signatures lorsque l'on s'écarte de la cote dipolaire revêt le caractère d'une règle générale dès que le dipôle horizontal présente une amplitude notable (dans notre exemple, il est six fois plus important pour le moins que le dipôle vertical).

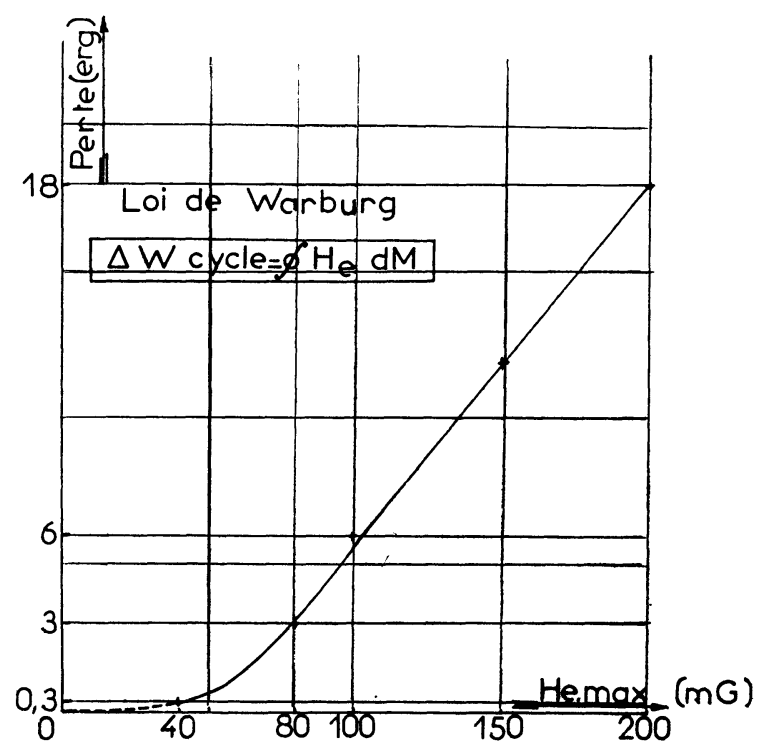

GRAPHIQUE DES PERTES D'UN BARREAU

RÉPARTITION DES PERTES MOYENNES PAR CYCLE POUR UN DÉPOINTAGE DE L'ORDRE DE $10^{\circ}$ $\left(H_{\mathrm{e}} \max \simeq 40 \mathrm{mG}\right)$

\begin{tabular}{ccc}
\cline { 2 - 3 } NOMBRE & $\begin{array}{c}\text { PERTE: } \\
\text { MAGNÉ- }\end{array}$ & $\begin{array}{c}\text { RAPPORT } \\
\text { D'INTER- } \\
\text { ACTION }\end{array}$ \\
TIQUE & - & - \\
1 & 0,31 erg & 1 \\
3 & 0,87 & 0,935 \\
5 & 1,40 & 0,905 \\
10 & 2,7 & 0,87 \\
$20+2$ aimants & 4,8 & 0,775 \\
\hline
\end{tabular}

FIG. 3. - Stabilisation d'attitude du satellite ESRO1 par hystérésis magnétique.

\section{CARACTÉRISTIQUES GÉOMÉTRIQUES :}

Cote du doublet : confondue avec la cote du centre de volume à $2 \mathrm{~cm}$ près.

Distance du doublet à l'axe vertical de symétrie : $8,2 \pm 1 \mathrm{~cm}$.

\section{CARACTÉRISTIQUES MAGNÉTIQUES :}

Dipôle vertical :

Moment permanent $31 \pm 4$ C.G.S.

Moment induit dans le champ terrestre très faible.

Dipôle horizontal :

Moment permanent $60 \pm 10$ G.G.S.

Moment induit dans le champ terrestre $120 \pm 20$ C.G.S.

Circuiterie :

Moment résultant de la mise sous tension des circuits $60 \pm 15$ G.G.S. 
La figure 2 présente l'image magnétique du satellite $\mathrm{D} 1$ telle qu'elle résulte des tests effectués selon la méthode exposée. On notera la précision médiocre à peine meilleure que $17 \%$, du fait du faible nombre d'observations effectuées sans montage différentiel.

Sur la figure 3, enfin, relative à la stabilisation d'attitude du satellite ESRO1, on peut voir d'une part l'évolution des pertes par hystérésis d'un barreau seul en fonction du champ externe, d'autre part la répartition des pertes résultant d'un dépointage de $10^{\circ}$ pour différentes configurations. Ge qui frappe, c'est la précision obtenue, meilleure que $8 \%$, alors que nous sommes tributaires de l'évaluation d'une série de moments magnétiques. Une telle précision n'a pu être atteinte qu'en multipliant les mesures et les conditions géométriques d'observation, afin de disposer de recoupements suffisants. 